\title{
Propagation of Coherent Light Pulses with PHASE
}

\author{
J. Bahrdt*a U. Flechsig ${ }^{\mathrm{b}}$, W. Grizollic ${ }^{\mathrm{c}}$, F. Siewert ${ }^{\mathrm{a}}$ \\ ${ }^{a}$ Helmholtz-Zentrum Berlin, Albert-Einstein-Straße 15, 12489 Berlin \\ ${ }^{\mathrm{b}}$ Paul-Scherrer-Institut, 5232 Villigen PSI, Switzerland \\ 'MAX IV Laboratory, Lund University, P.O. Box 118, SE-22100 Lund, Sweden
}

\begin{abstract}
The current status of the software package PHASE for the propagation of coherent light pulses along a synchrotron radiation beamline is presented. PHASE is based on an asymptotic expansion of the Fresnel-Kirchhoff integral (stationary phase approximation) which is usually truncated at the $2^{\text {nd }}$ order. The limits of this approximation as well as possible extensions to higher orders are discussed. The accuracy is benchmarked against a direct integration of the Fresnel-Kirchhoff integral. Long range slope errors of optical elements can be included by means of $8^{\text {th }}$ order polynomials in the optical element coordinates $w$ and $l$. Only recently, a method for the description of short range slope errors has been implemented. The accuracy of this method is evaluated and examples for realistic slope errors are given. PHASE can be run either from a built-in graphical user interface or from any script language. The latter method provides substantial flexibility. Optical elements including apertures can be combined. Complete wave packages can be propagated, as well. Fourier propagators are included in the package, thus, the user may choose between a variety of propagators. Several means to speed up the computation time were tested - among them are the parallelization in a multi core environment and the parallelization on a cluster.
\end{abstract}

Keywords: physical optics, propagation of coherent light

\section{INTRODUCTION}

In the past synchrotron radiation beamlines have been optimized utilizing ray tracing codes such as SHADOW [1] [2] or RAY [3]. With the advent of $3^{\text {rd }}$ generation storage rings and diffraction limited $4^{\text {th }}$ generation light sources such as Xray Free Electron Lasers (FEL) [4] [5] and Diffraction Limited Storage Rings (DLSR), e.g. [6] more advanced tools have to be used. Generally, the existing codes are either based on Fourier Optics (FO), e.g. SRW, or on the evaluation of the Fresnel-Kirchhoff integral by means of the Stationary Phase Approximation (SPA). Though time consuming, a numeric integration of the integral is successfully used for benchmarking. We call the method FKOE which indicates the integration of the Fresnel-Kirchhoff integral including an OE. In this article we concentrate on the SPA-method. The algorithm is implemented in the software package PHASE which has been developed over many years at BESSY / HZB.

PHASE is based on power series expansions of the optical path length function. Analytic expressions for the principle rays and the related phase and the two-dimensional integrals over the OEs are derived. The method is described in [7] [8] [9]. Earlier versions were based on expressions derived with the algebraic code REDUCE which produced a huge source code and, thus it was limited to the $5^{\text {th }}$ order expansion of the OE surface shape. A new Ansatz permits a compact formulation of the equations enabling an expansion up to $8^{\text {th }}$ order and higher [10]. The new algorithm was implemented in PHASE three years ago.

In section 2 the $2^{\text {nd }}$ order stationary phase approximation is described and the limitations are discussed. Generally, the accuracy of the results depends strongly on the chosen geometry, i.e. the position of the Source Plane (SP) and the Image Plane (IP). The geometry can always be formulated in a manner which minimizes the errors related to the $2^{\text {nd }}$ order SPAmethod. On the other hand, an extension of the asymptotic expansion of the Fresnel-Kirchhoff integral is obvious from literature and an implementation into PHASE is possible.

The PHASE package consists of several program versions which are adapted to specific tasks. Additionally, many routines such as Fourier propagators are available. Section 3 reviews the current status of the complete PHASE package.

Short range OE slope errors are limiting the performance of high end beamlines. In section 4 we present a new method for the simulation of slope errors within PHASE. The accuracy is discussed and examples are given. 


\section{PROPAGATION USING $2^{\text {ND }}$ ORDER STATIONARY PHASE APPROXIMATION}

\section{$2^{\text {nd }}$ Order Approximation of Path Length}

Let us assume a simple geometry with one optical element, an electric field distribution in a source plane and an image plane where the electric field distribution is to be evaluated (Fig.1). Following the SPA-method it is important to avoid geometries where SP and IP both are focal planes, i.e. planes with constant phase. Otherwise, the expressions diverge due to division by zero. Usually, the source plane is chosen not to be a focal plane. This does not limit the generality of the approach, because a source with a flat wavefront can always be propagated downstream towards the next OE. The PHASE package includes FO-propagators for this purpose. Different propagators for the near-field and the far-field as well as for the Fraunhofer limit are implemented.

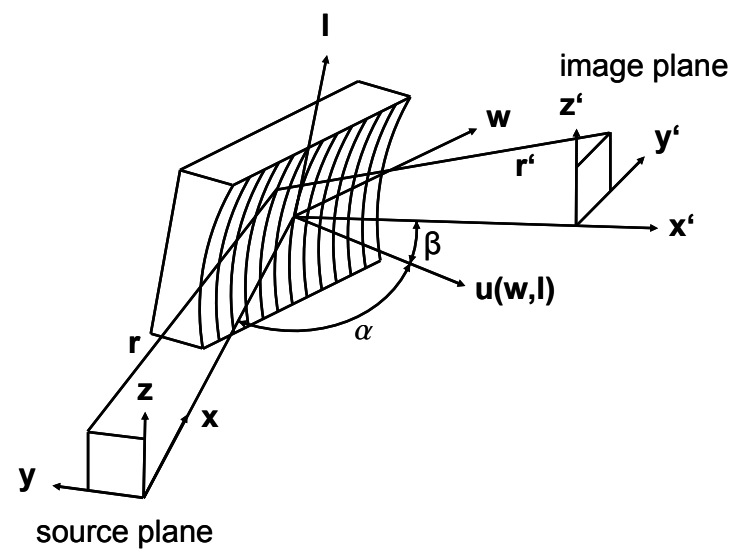

Figure 1: One optical element between a source plane and an image plane.

The field distribution of a coherent beam as propagated across an OE is given by the Fresnel-Kirchhoff integral (Eq.1). The outer double integral is evaluated over the source plane. The double integral in the bracket is evaluated over the optical element surface.

$$
E\left(y^{\prime}, z^{\prime}\right)=\frac{\sqrt{\cos (\alpha)} \sqrt{\cos (\beta)}}{\lambda^{2}} \iint E(y, z)\left\{\iint \frac{1}{r \cdot r^{\prime}} e^{i k P L} d w \cdot d l\right\} d y \cdot d z
$$

The integration over $(y, z)$ in the source plane can be replaced by an integration over angles (dy',dz') in the image plane introducing an appropriate functional determinant:

$$
E\left(y^{\prime}, z^{\prime}\right)=\frac{\sqrt{\cos (\alpha)} \sqrt{\cos (\beta)}}{\lambda^{2}} \iint E(y, z)\left\{\iint \frac{1}{r \cdot r^{\prime}} e^{i k P L} d w \cdot d l\right\}\left|\frac{\partial(y, z)}{\partial\left(d y^{\prime}, d z^{\prime}\right)}\right| d d y^{\prime} \cdot d d z^{\prime}
$$

Eq.2 may be evaluated directly (FKOE method) which can be pretty time-consuming. Nevertheless, it serves as a valuable reference for benchmarking. The tricky part is an analytic integration of the double integral in brackets and we will concentrate on this term. Following Mandel and Wolf [11] we call this term $F_{2}(k)$ where the subscript 2 is related to the $2^{\text {nd }}$ order expansion of the optical path length PL:

$$
F_{2}(k)=\iint \frac{1}{r \cdot r^{\prime}} e^{i k P L} d w \cdot d l
$$

$r, r^{\prime}, P L$ depend upon the initial coordinates $(y, z)$ and the final coordinates $\left(y^{\prime}, z^{\prime}\right)$. For the moment, we assume that the finite dimensions of the optical element do not contribute to the integral (e.g. no diffraction at the edges). Then, the double integral can be evaluated analytically on the interval $(-\infty,+\infty)$. The principle rays across an OE are defined by the critical points of the $1^{\text {st }}$ kind, i.e. the points at the OE where the $1^{\text {st }}$ partial derivatives of PL are zero. Around the critical points (CPs) of the $1^{\text {st }}$ kind $\left(w_{0}, l_{0}\right)$ PL can be expanded with respect to $w$ and $l$ :

$$
P L\left(w_{0}+\Delta w, l_{0}+\Delta l\right)=P L_{w_{0} l_{0}}+\left.\frac{1}{2} \frac{\partial^{2} P L}{\partial w^{2}}\right|_{w_{0} l_{0}} \Delta w^{2}+\left.\frac{1}{2} \frac{\partial^{2} P L}{\partial l^{2}}\right|_{w_{0} l_{0}} \Delta l^{2}+\left.\frac{\partial^{2} P L}{\partial w \cdot \partial l}\right|_{w_{0} l_{0}} \Delta w \cdot \Delta l
$$


In the following we will skip the $\Delta$ assuming that $w$ and $l$ are always taken with respect to $w_{0}$ and $l_{0}$ (rather than $(0,0)$ ).

$$
P L=P L_{w_{0} l_{0}}+\frac{1}{2} P L_{w w} \cdot w^{2}+\frac{1}{2} P L_{l l} \cdot l^{2}+P L_{w l} \cdot w \cdot l=P L_{w_{0} l_{0}}+a \cdot w^{2}+b \cdot l^{2}+c \cdot w \cdot l
$$

Assuming $r, r$ ' to be independent upon the location of the $\mathrm{CP}$ an analytic integration over the $\mathrm{OE}$ coordinates yields Eq.6

$$
F_{2}(k) \cong \frac{1}{r_{w_{0} l_{0}} \cdot r^{\prime}{ }_{w_{0} l_{0}}} e^{i k P L_{w_{0} l_{0}}} \frac{i j \lambda}{\sqrt{\left|P L_{w w} P L_{l l}-P L_{w l} P L_{w l}\right|}}
$$

where $j$ is defined as:

$$
\begin{array}{clcc}
1 & \text { if } & P L_{w w} P L_{l l}-P L_{w l}^{2}>0 \wedge P L_{w w}>0 \\
j=-1 & \text { if } & P L_{w w} P L_{l l}-P L_{w l}^{2}>0 \wedge P L_{w w}<0 \\
i & \text { if } & P L_{w w} P L_{l l}-P L_{w l}^{2}<0
\end{array}
$$

With an orthogonal coordinate transformation the cross term can be removed and the double integral splits into two individual integrals which are integrated separately. The coordinate transformation can be performed in various manners. Two of them are given in Eq.7 and Eq.8:

$$
\begin{aligned}
& \widetilde{w}=w \quad \tilde{l}=l+\frac{c}{2 b} w \\
& \widetilde{w}=w+\frac{c}{2 a} l^{2} \quad \tilde{l}=l
\end{aligned}
$$

As a result we get the integral in the new coordinates [11]:

$$
\begin{aligned}
& F_{2}(k) \cong \frac{1}{r_{w_{0} l_{0}} \cdot r^{\prime}{ }_{w_{0} l_{0}}} e^{i k P L_{w_{0} l_{0}}} \frac{\exp _{P L_{\tilde{w} \tilde{w}}}\left( \pm i \cdot \frac{\pi}{4}\right) \cdot \exp _{P L_{\tilde{l}}}\left( \pm i \cdot \frac{\pi}{4}\right) \cdot \lambda}{\sqrt{\left|P L_{\widetilde{w} \widetilde{w}} P L_{\tilde{l} \tilde{l}}\right|}} \\
& +\quad \text { if } \quad P L_{\tilde{w} \widetilde{w}}\left(P L_{\tilde{l} \tilde{l}}\right)>0 \\
& -\quad \text { if } \quad P L_{\tilde{w} \widetilde{w}}\left(P L_{\tilde{l} \tilde{l}}\right)<0
\end{aligned}
$$

Power series expansions of the square root argument in Eq.6, Eq.9 \& Eq.7 und Eq.9 \& Eq.8 are implemented in PHASE. Mathematically, the three expressions, Eq.6, Eq.9 \& Eq.7 and Eq.9 \& Eq.8 are equivalent. However, they differ in the convergence of the power series expansion and in the numerical noise behavior as will be demonstrated with the following reference geometry: A toroidal mirror operated at $2^{\circ}$ grazing incidence de-magnifies the source by a factor of 30 . The source is a $10 \mathrm{~nm}$ wavelength Gaussian beam with a waist radius of $0.07 \mathrm{~mm}$. The distances are: source waist $\mathrm{OE}=15000 \mathrm{~mm} ; \mathrm{OE}-$ focal plane $=500 \mathrm{~mm}$. The electric field distribution is defined in the source plane. In the example it is located $5000 \mathrm{~mm}$ downstream of the source waist. In the source plane the radius of the Gaussian beam is grown to $0.238 \mathrm{~mm}$ and the wavefront is spherical. Fig. 2 left shows the exact intensity distribution in the image (focal) plane as evaluated with the FKOE method. Fig.2 right was evaluated in the $0^{\text {th }}$ order SPA which is limited to the evaluation of the principle rays, only, where individual phases are attributed to the principle rays. This approach goes beyond the Ansatz of Schäfers [3], since the source is defined by a diffraction limited electric field distribution. A comparison with Fig.2 left demonstrates the low accuracy of the $0^{\text {th }}$ order SPA. Higher order terms have to be included.

The $1^{\text {st }}$ order terms of the SPA are zero by definition, and the $2^{\text {nd }}$ order approximation is utilized in most cases. In Fig. 3 several versions of the $2^{\text {nd }}$ order SPA are compared. Eq. 6 describes the correct focus shape. However, the intensity is off by about $20 \%$ (Fig.3 left). The discrepancy from the FKOE result is due to an interference of neighboring CPs in this specific geometry. An integration to infinity for each individual CPs does not represent a precise integration of the interfering pattern. The results based on Eq.9\&7 or Eq.9\&8 are even worse. This is caused by the bad convergence of the Taylor series expansion of the expressions in Eq.7 and Eq.8. The convergence is still better for Eq.7 due to the steep curvature of the $\mathrm{OE}\left(2^{\text {nd }}\right.$ derivative of $\left.\mathrm{PL}\right)$ in transverse direction in this specific case. 

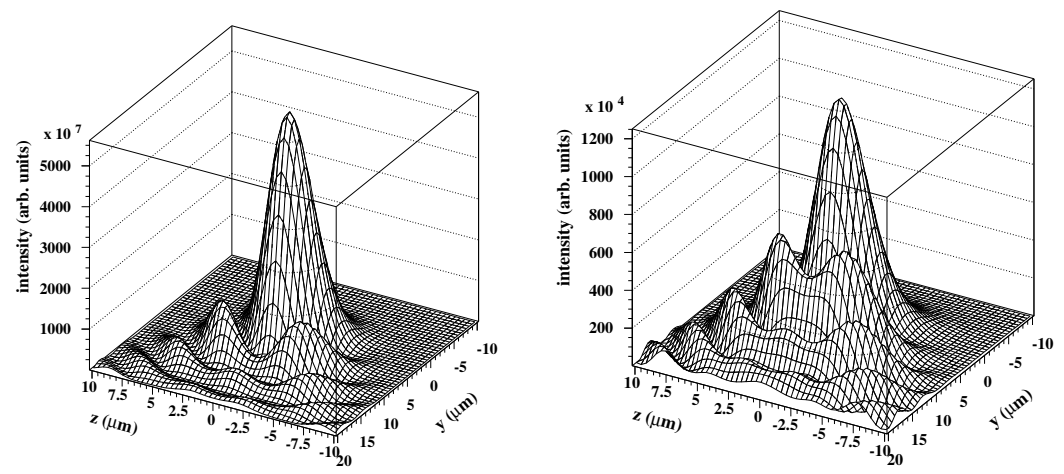

Figure 2: Reference geometry as described in the text; $\mathrm{SP}=5000 \mathrm{~mm}$ (distance to source waist); number of critical points (CPs) on the OE surface: $601 \times 601$. Left: FKOE. Right: $0^{\text {th }}$ order SPA.
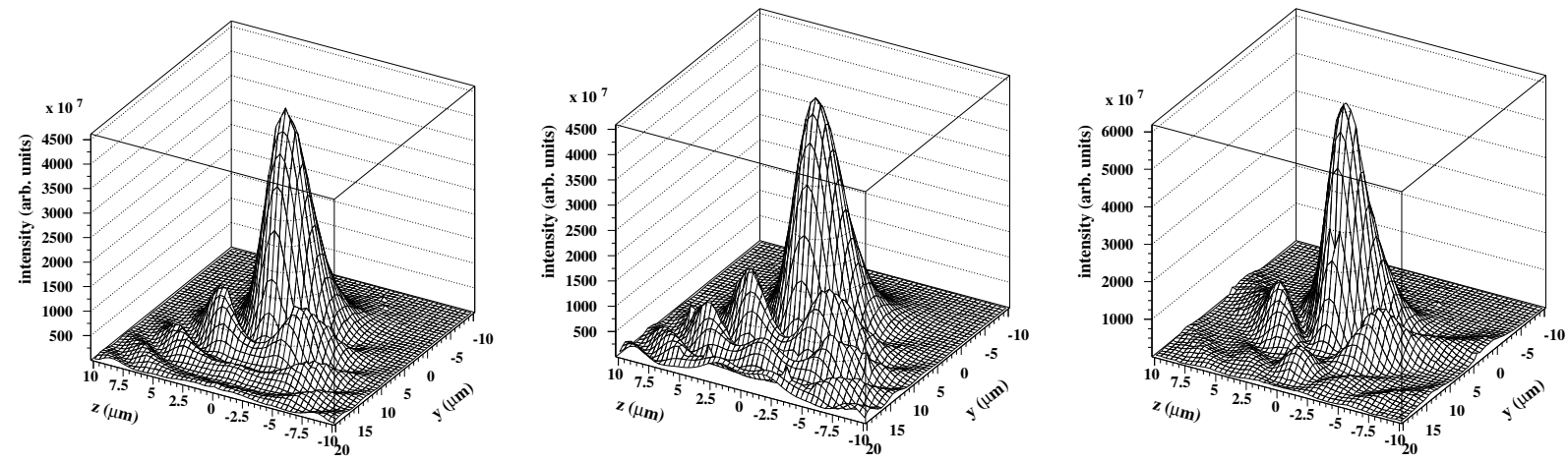

Figure 3: Reference geometry; SP=5000mm; $601 \times 601$ CPs. Various methods of $2^{\text {nd }}$ order SPA are applied: Left: $2^{\text {nd }}$ order SPA, Eq.6; center: $2^{\text {nd }}$ order SPA, Eq. 9 \& transformation 7; right: $2^{\text {nd }}$ order SPA, Eq. 9 \& transformation 8.

If the number of critical points at the OE surface is reduced the numeric noise increases (Fig.4). The sharp spikes result from small $2^{\text {nd }}$ derivatives of PL in the denominator. Obviously, the singularities are sampled more accurately with a finer mesh as demonstrated in Fig.3. The spikes are most prominent when utilizing Eq.6 whereas they are damped a bit with the Taylor series expansions in Eq.7 and Eq8. Fig.4 suggests the use the Eq.9 with transformation Eq.7 for an efficient suppression of the noise with a small number of CPs.
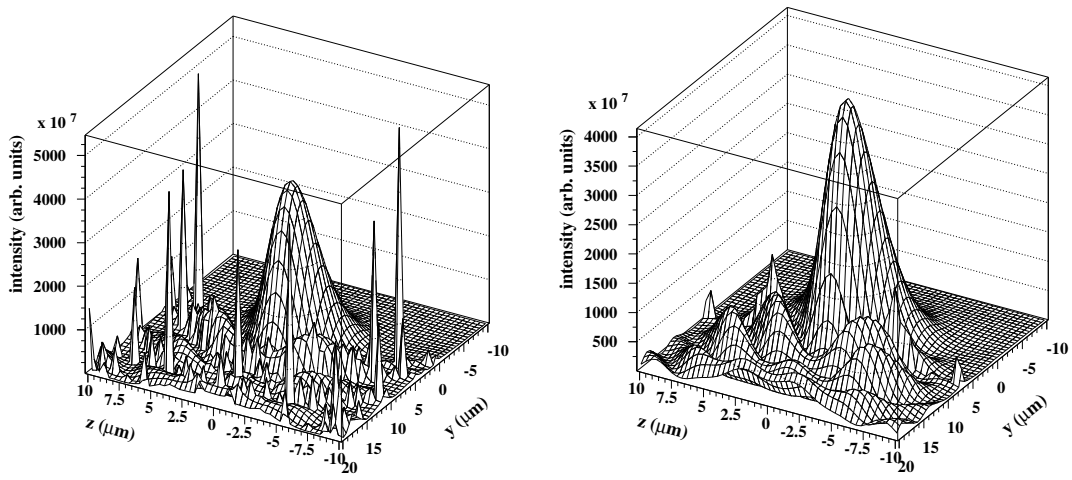

Figure 4: Reference geometry; SP=5000mm; 41 x 41 CPs; left: Propagation based on Eq.6; right: Propagation based on Eq.9 with transformation Eq.7. 
The interference of critical points can be reduced efficiently when the source plane is shifted further from the source waist away towards the OE. The numeric noise is reduced as well. Fig.5 left and center are evaluated with the same number of CPs as Fig4 left and right, however, the total intensity is now closer to the FKOE result and the spikes are suppressed. Interestingly, the $0^{\text {th }}$ order SPA delivers more accurate results, as well (Fig.5 right).

Thus, the appropriate choice of the source plane is an essential issue for high computation speed and accurate results. A further reduction of the number of CPs is possible with a non-equidistant grid of CPs where the density has to be increased close to small $2^{\text {nd }}$ derivatives of PL (not implemented in PHASE, yet).
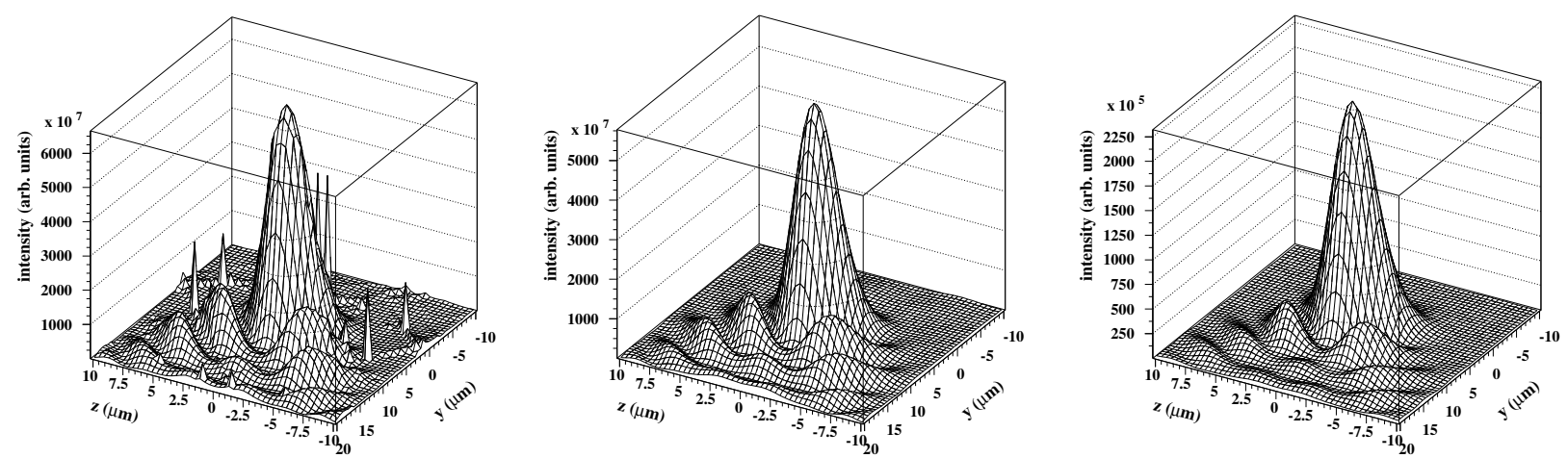

Figure 5: OE and image plane as in reference case; source plane moved to SP $=10000 \mathrm{~mm}$; 41 x 41 CPs. Left: Eq.6; center: Eq.9 with transformation Eq.7; right: $0^{\text {th }}$ order SPA.

The FKOE method which we use for benchmarking is implemented as a two-step algorithm for the evaluation of the integral in Eq.1: i) propagation from the source plane to the OE; ii) propagation from the OE to the image plane. In both steps the CPU time is defined by a 4-dimensional sum (in contrast to the 6-dim sum of Eq.1), and, thus, the CPU time seems to be comparable to the PHASE algorithm. The phase advance of the electric field at the intermediate plane, the OE surface, is extremely high. Nevertheless, surprisingly good results are achieved if the image plane is a bi-focal plane and, thus, the FKOE method is a valuable tool for benchmarking SPA. Interestingly, the FKOE method permits an elegant implementation of slope errors and apertures at the OE surface in this case. Unfortunately, out of focus the method is not adequate because the CPU time increases dramatically due to the required refinement of the grid at the OE surface which is not needed in PHASE. Even an enhancement of the grid points of an order of magnitude in both directions provides worse results than $2^{\text {nd }}$ order SPA (Fig. 6). In specific cases one may propagate with the FK-method to an intermediate focus and, then, propagate the beam back via Fourier optics. Mostly, however, intermediate bi-focal planes do not exist.
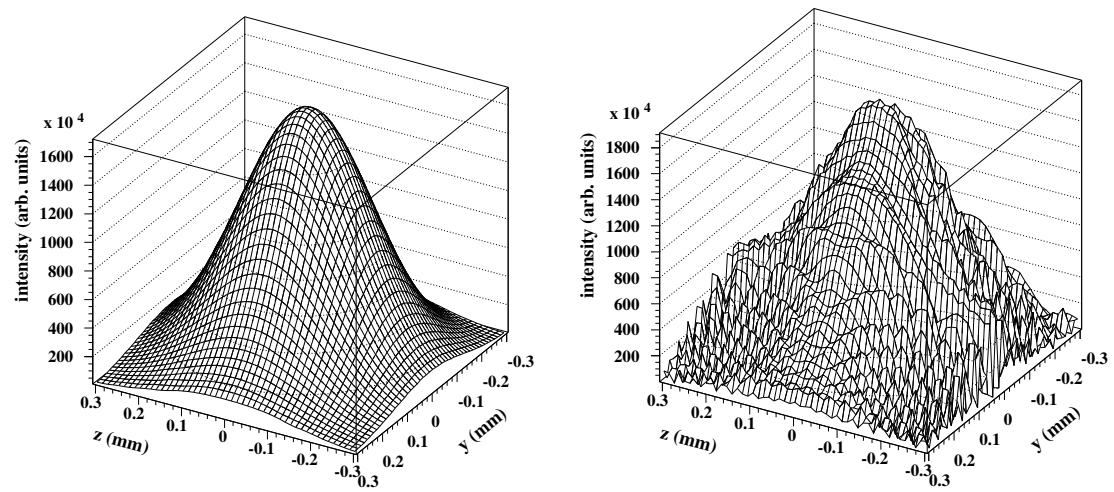

Figure 6: Source plane and OE as in reference geometry; image plane moved 200mm upstream with respect to the focal plane. Left: $2^{\text {nd }}$ order SPA with $61 \times 61$ critical points; right: FKOE with $601 \times 601$ data points on the optical element surface. 


\section{Limits of Second Order Expansion}

In extreme cases the interference of neighboring critical points determines the accuracy of the $2^{\text {nd }}$ order approximation which is illustrated in Fig.7 where the contribution from a critical point at $w_{0} \approx 17 \mathrm{~mm}$ is studied. Obviously, this CP interferes with another $\mathrm{CP}$ at $w_{0} \approx-30 \mathrm{~mm}$ which degrades the precision. The results may be improved with the implementation of a $3^{\text {rd }}$ order term in PL. As described in [10] with a suited coordinate transformation the integral of Eq. 3 can be written in the form of Eq.10 ( $k$ is integrated in the new factors $\tilde{a}$ and $\tilde{b})$.

$$
F_{2+}(k) \cong \frac{1}{r_{w_{0} l_{0}} \cdot r^{\prime}{ }_{w_{0} l_{0}}} e^{i k P L_{w_{0} l_{0}}} \tilde{a} \int e^{i\left(\widetilde{w}^{3}+\tilde{b} \widetilde{w}^{2}\right)} d \widetilde{w} \cdot \int e^{i \cdot \tilde{l}^{2}}
$$

Then, the integral including the third order terms can be integrated analytically:

$$
\begin{aligned}
\tilde{a} \int e^{i\left(\widetilde{w}^{3}+\tilde{b} \widetilde{w}^{2}\right)} d \widetilde{w} & \approx \frac{2 \tilde{a}}{3} \sum_{k=0,2,4 \ldots}^{\infty} \frac{\tilde{b}^{k}}{k !} \Gamma\left(\frac{2 k+1}{3}\right) \cos \left(\frac{1-k}{6} \pi\right) \\
& -\frac{2 \tilde{a}}{3} i \sum_{k=1,3,5 \ldots}^{\infty} \frac{\tilde{b}^{k}}{k !} \Gamma\left(\frac{2 k+1}{3}\right) \sin \left(\frac{1-k}{6} \pi\right)
\end{aligned}
$$
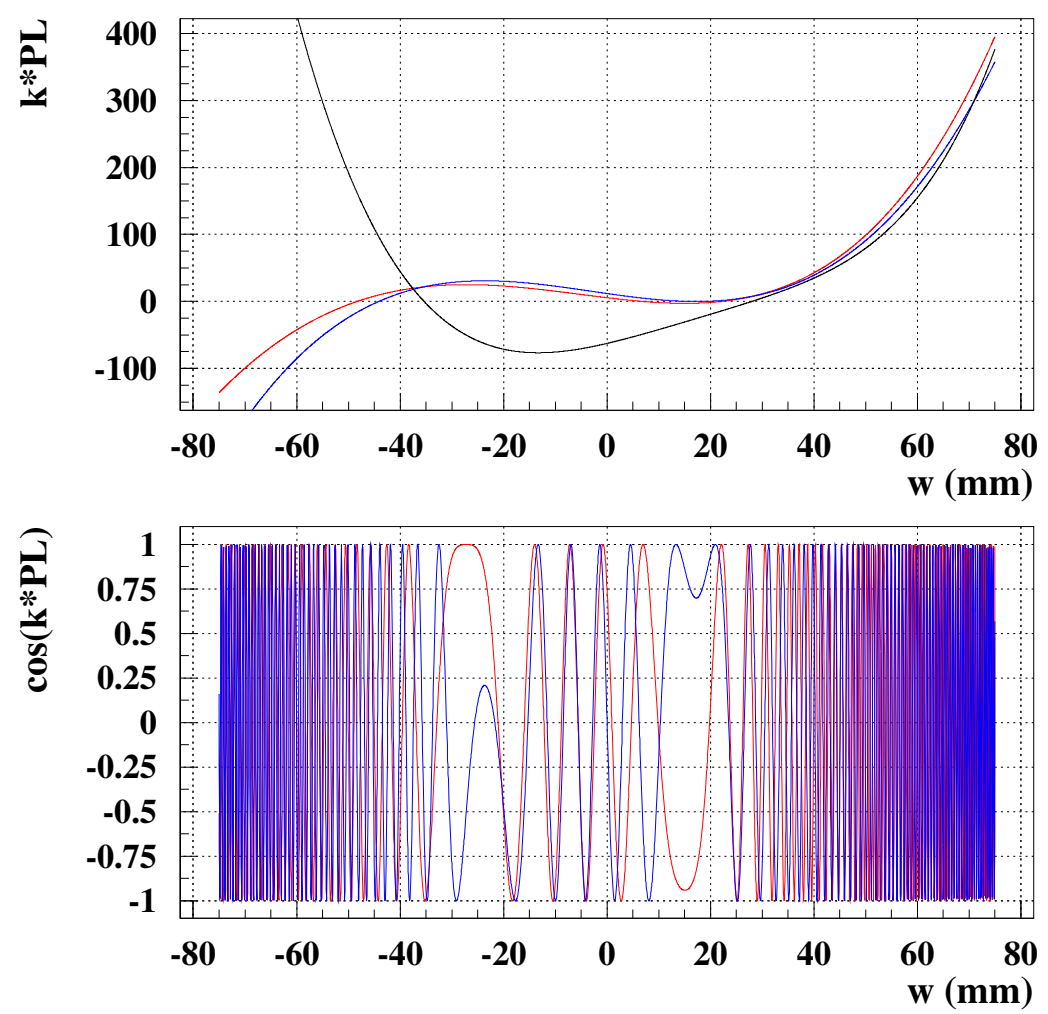

Figure 7: Top: Normalized optical path exactly evaluated (red) and approximated to $3^{\text {rd }}$ order in $w$ and $2^{\text {nd }}$ order in $l$ (blue). The black line is the difference multiplied by a factor of 10. Bottom: cos-function of the normalized optical paths (colors as above).

Two equivalent methods for the evaluation of the $1^{\text {st }}$ integral in Eq.10 are implemented in PHASE: i) direct evaluation of Eq.11; ii) interpolation utilizing a table which has been generated with an analytic integration with Mathematica. The cos- and sin-terms of the integral are plotted in Fig.8. The applicability and accuracy of this method is under investigation. For our reference case it does not improve the results of the $2^{\text {nd }}$ order SPA because only small errors (represented by the residuals in Fig.7 top) change the phase substantially and, hence, the contributions to the integral 
from interfering CPs at large distances are encounter large errors. Instead of integrating over two CPs in a single step it may be better to include $3^{\text {rd }}$ order terms of the asymptotic expansion for each individual $\mathrm{CP}$. Though not yet implemented in PHASE, the relevant terms of the complete asymptotic expansion are adressed in the next chapter.

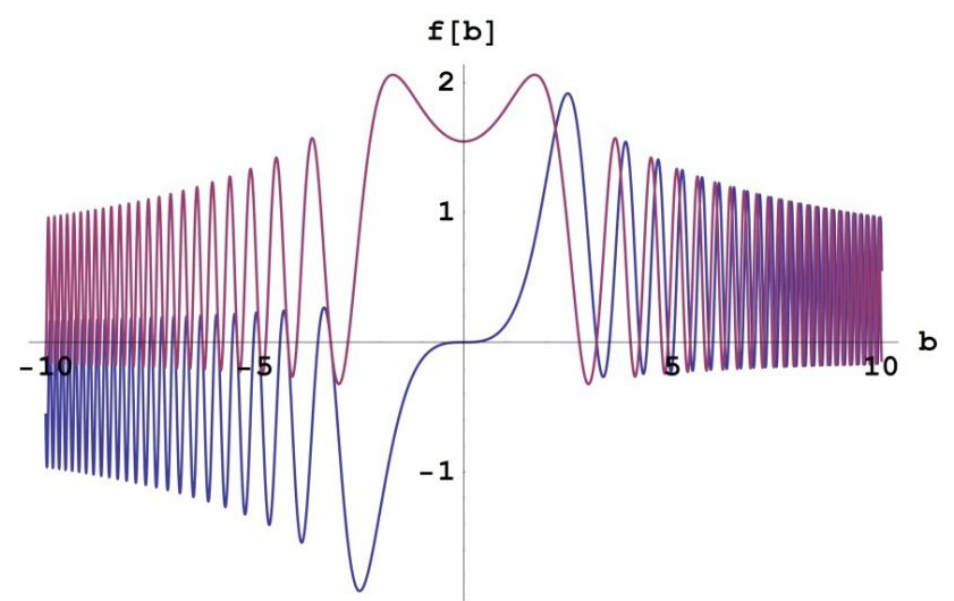

Figure 8: Integration of the cos-part (red) and the sin-part (blue) of the $1^{\text {st }}$ integral in Eq.10 using Mathematica. The results are identical to the result from the series expansion of Eq.11. The x-coordinate corresponds to $\tilde{b}$ in Eq.11.

\section{Complete asymptotic expansion an application to PHASE}

Focke presents a complete asymptotic expansion of the Fresnel-Kirchhoff integral [12]. He presents the formalism to solve the integral in many specific cases, e.g.:

- critical points of the $1^{\text {st }}$ kind: points on the $\mathrm{OE}$ with zero derivatives

- critical points of the $2^{\text {nd }}$ kind: points on the boundary with zero derivative along the boundary

- critical points of the $3^{\text {rd }}$ kind: edges on the boundary

We briefly recall Focke's equations for the critical points of the $1^{\text {st }}$ kind and comment on the applicability to PHASE. We skip the critical points of the $2^{\text {nd }}$ kind because apertures can be treated separately as individual elements located close to the OEs.

In a first step the cross term in the expansion of PL must be removed. The quadratic form can be transformed to principle axis with an orthogonal transformation.

$$
\begin{gathered}
w=w_{0}+a \cdot h+b \cdot k \\
l=l_{0}+c \cdot h+d \cdot k
\end{gathered}
$$

The transformation matrix to the new coordinates $(h, k)$ has a determinant of 1 . The form is not unique and must be chosen appropriately for low numeric noise. The number small depends upon the specific geometry and grid size.

$$
\begin{aligned}
\left(\begin{array}{ll}
a & b \\
c & d
\end{array}\right) & =\left(\begin{array}{cc}
1 & -P L_{w l} / P L_{w w} \\
0 & 1
\end{array}\right) \text { if }\left|P L_{w w}\right|>\text { small } \\
\left(\begin{array}{ll}
a & b \\
c & d
\end{array}\right) & =\left(\begin{array}{cc}
1 & 0 \\
-P L_{w l} / P L_{l l} & 1
\end{array}\right) \text { if }\left|P L_{l l}\right|>\text { small } \\
\left(\begin{array}{ll}
a & b \\
c & d
\end{array}\right) & =\frac{1}{2}\left(\begin{array}{cc}
1 & 1 \\
-1 & 1
\end{array}\right) \text { if }\left|P L_{w w}\right|<\text { small } \wedge\left|P L_{l l}\right|<\text { small }
\end{aligned}
$$

The partial derivatives of the path length in new coordinates are abbreviated with

$$
P L_{\mu, v-\mu}=\frac{1}{\mu !(v-\mu) !} \frac{\partial^{v} P L}{\partial^{\mu} h \partial^{v-\mu} k}
$$


Following the philosophy of PHASE the orthogonal transformation and the evaluation of the partial derivatives of PL has to be done only once for a given geometry. Having the Taylor series expansions of the partial derivatives in terms of $\left(y^{\prime}, z^{\prime}, d y^{\prime}, d z^{\prime}\right)$ the complete asymptotic expansion of the FK-Integral is given by the sum over the contribution from all critical points. With the substitutions $\varphi_{\text {Focke }}=-P L$ and $g_{\text {Focke }}=1 / r r^{\prime}$ we get the contribution from the CP $\left(w_{0}, l_{0}\right)$ from Eq.15, Eq.16 and Eq.17 [12].

$$
\begin{aligned}
& I_{w_{0} l_{0}}=e^{i k P L_{w_{0} l_{0}}} \frac{2 \pi i j}{\sqrt{\left|P L_{w w} P L_{l l}-P L_{w l}^{2}\right|}} \cdot \sum_{n=0}^{\infty} A_{n} \frac{1}{i}\left(\frac{1}{i k}\right)^{n+1} \\
& A_{n}=\sum_{\tau=0}^{2 n} \sum_{s+q=n+\tau} \frac{s ! ! q ! !}{\left(2 \cdot P L_{2,0}\right)^{s}\left(2 \cdot P L_{0,2}\right)^{q}} \sum_{\substack{\sigma=0 \ldots 2 s \\
\rho=0 \ldots 2 q \\
\sigma+\rho \geq 3 \tau}}\left(\frac{1}{r r^{\prime}}\right)_{2 s-\sigma, 2 q-\rho} \Phi_{\tau, \sigma, \rho} \\
& \phi_{\tau, \sigma, \rho}=(-1)^{\tau} \sum_{\substack{\gamma_{30}+\gamma_{21}+\cdots+\gamma_{0, \sigma+\rho}=\tau \\
3 \gamma_{30}+2 \gamma_{21}+\cdots+0 \gamma_{0, \sigma+\rho}=\sigma \\
0 \gamma_{30}+1 \gamma_{21}+\cdots+(\sigma+\varrho) \gamma_{0, \sigma+\rho}=\rho}} \frac{P L_{30}^{\gamma_{30}} \cdot P L_{21}^{\gamma_{21}} \cdot P L_{12}^{\gamma_{12}} \cdot P L_{03}^{\gamma_{03} \ldots P L_{0, \sigma+\rho}}}{\gamma_{30} ! \gamma_{21} ! \gamma_{12} ! \gamma_{03} ! \ldots \gamma_{0, \sigma+\rho} !}
\end{aligned}
$$

All non-addressed $\Phi_{\tau, \sigma, \varrho}$ are zero.

For $n=0$ we get $A_{0}=1$ and the result is the well-known $2^{\text {nd }}$ order stationary phase approximation (Eq.6). The next order terms are derived from $A_{1}=A_{1 a}+A_{1 b} . A_{1 a}$ (Eq. 18) summarizes the terms of $A_{1}$ with the approximation of $g_{\text {Focke }}=1 / r r^{\prime}=$ const and $\frac{\partial^{v} g_{\text {Focke }}}{\partial h^{\mu} \partial k^{\nu-\mu}}=0$ for $v>0$ :

$$
\begin{aligned}
A_{1 a}=\frac{1}{r r^{\prime}}[ & -\frac{1}{\left(2 \cdot P L_{20}\right)^{2}} \cdot \frac{P L_{40}}{1}-\frac{1}{\left(2 \cdot P L_{20}\right)\left(2 \cdot P L_{02}\right)} \cdot \frac{P L_{22}}{1}-\frac{1}{\left(2 \cdot P L_{02}\right)^{2}} \cdot \frac{P L_{04}}{1}+ \\
& \frac{3}{\left(2 \cdot P L_{20}\right)^{3}} \cdot \frac{P L_{30}^{2}}{1}+\frac{3}{\left(2 \cdot P L_{02}\right)^{3}} \cdot \frac{P L_{03}^{2}}{2}+ \\
& \frac{1}{\left(2 \cdot P L_{20}\right)^{2}\left(2 \cdot P L_{02}\right)} \cdot \frac{P L_{21}^{2}}{2}+\frac{1}{\left(2 \cdot P L_{20}\right)\left(2 \cdot P L_{02}\right)^{2}} \cdot \frac{P L_{12}^{2}}{2}+ \\
& \left.\frac{1}{\left(2 \cdot P L_{20}\right)^{2}\left(2 \cdot P L_{02}\right)} \cdot \frac{P L_{30} P L_{12}}{1}+\frac{1}{\left(2 \cdot P L_{20}\right)\left(2 \cdot P L_{02}\right)^{2}} \cdot \frac{P L_{21} P L_{03}}{1}\right]
\end{aligned}
$$

The first three terms of $A_{1 a}$ are evaluated for $\tau=1 \wedge \sigma+\rho=4$ and the next six terms for $\tau=2 \wedge \sigma+\rho=6$. Including the dependence of $1 / r r^{\prime}$ on $h$ and $k$ six additional terms show up which are abbreviated with $A_{1 b}$ (Eq.19):

$$
\begin{aligned}
A_{1 b}= & \frac{\partial^{2}}{\partial h^{2}}\left(\frac{1}{r r^{\prime}}\right)+\frac{\partial^{2}}{\partial k^{2}}\left(\frac{1}{r r^{\prime}}\right)+ \\
& \frac{\partial}{\partial h}\left(\frac{1}{r r^{\prime}}\right)\left[-\frac{1}{\left(2 \cdot P L_{20}\right)\left(2 \cdot P L_{02}\right)} \cdot \frac{P L_{12}}{1}-\frac{1}{\left(2 \cdot P L_{20}\right)^{2}} \cdot \frac{P L_{30}}{1}\right]+ \\
& \frac{\partial}{\partial k}\left(\frac{1}{r r^{\prime}}\right)\left[-\frac{1}{\left(2 \cdot P L_{20}\right)\left(2 \cdot P L_{02}\right)} \cdot \frac{P L_{21}}{1}-\frac{1}{\left(2 \cdot P L_{02}\right)^{2}} \cdot \frac{P L_{02}}{1}\right]
\end{aligned}
$$

Analytic expressions of $P L, r, r$ ' are already implemented in PHASE and their partial derivatives can easily be derived. Thus, the coding of $A_{1}$ is straight forward. The influence of the $3^{\text {rd }}$ order derivatives on the accuracy will be subject of future studies.

\section{FEATURES AND IMPLEMENTATION DETAILS}

The PHASE program suite has been developed over the last 20 years from a proof of principle study on a VAX computing environment to an almost full featured ray tracing and physical optics package tailored to the specific 
requirements of synchrotron and Free Electron Laser (FEL) optics simulations running on any UNIX like operating system $^{1}$. It consists of various executable programs coded in $C++, C$ and Fortran 77 with VAX extensions. We also compiled an ISO image which can directly be booted from CD or USB stick without prior installation of Linux or can be executed on a virtual machine.

The main program is called phaseqt for interactive usage with a graphical user interface based on the modern $Q t^{2}$ widget set. Plotting of inputs and results is realized with the $Q w t^{3}$ plotting library. phasesrv and phasempi are slim versions of the program without graphical user interface. They have less dependencies to external libraries and can be installed on servers without root access. Additional programs are phaseopti, phaseextract, fkoe and fkoempi which provide beamline optimization ${ }^{4}$, detailed evaluation of aberrations and Fresnel- Kirchhoff integration.

PHASE takes full advantage of modern multi-core CPU's by executing the calculations in parallel. For physical optics calculations we define one task for each pixel in the image plane. The programs use different approaches: phaseqt uses the QtConcurrent API for multi-threaded programs. Internally QtConcurrent works with a $1 \mathrm{~d}$ task vector- in our case we use the index of the pixel to identify a task. QtConcurrent distributes the tasks to the available cores or threads until all tasks are finished. There is a very useful Pause, Resume and Abort functionality. The maximum number of threads can be chosen. This is particular important on multi-user machines. Without restriction QtConcurrent uses all threads which are available. phasesrv is based on POSIX threads. This approach divides the total number of pixels into packages before the calculation starts, each package becomes one task which is then executed by one thread. Typically the number of used threads is chosen equal to the number of packages. On a single user machine a much higher number of packages may result in faster execution since the computation time for the individual pixels can be quite different. phasempi uses the master slave approach of Open MPI ${ }^{5}$. It distributes the tasks on different nodes on a High Performance Computing (HPC) cluster. One host acts as the master and distributes the tasks from a task-vector to the slaves. At the beginning the slaves register at the master that they are ready to take any task, the master sends a message with the taskid (the pixel-number) to each slave. After the slave has finished his task it sends a message back to the master. The process continues until all tasks are finished. Usually for HPC there is a Resource Management System in place. It manages the number of nodes, scheduling, accounting, billing. Typically: a higher number of nodes executes faster but it may take longer to get scheduled.

The prominent feature of PHASE is the physical optics calculation based on the stationary phase approach. Some recent extensions in the physical optics mode are: (a) Considering the phase shift due to slope errors of real optics, given by a $2 \mathrm{~d}$ height error file, in the past we considered only ideal optics and some waviness. (b) Considering apertures and the usable size of optical elements, in the past the size was not constrained. (c) Considering coating materials via the atomic scattering factors [13] quantitative evaluation of intensities and polarization is now available. The current calculation takes the medium deflection angle of the optics as input, an extension to use the local slope is possible in principle. (d) Support of the $\mathrm{HDF}^{6}{ }^{6}$ file format for input and output allows easy data exchange with other programs like GENESIS [14]. (e) The grid size in the source and image plane is no longer limited by the program, just by the available dynamic memory. (f) Fourier optics propagators based on the fftw $3^{7}$ library have been added to phaseqt. This allows quick cross-checks and fast free-space propagation. A Transfer function (TR) based propagator (Fourier) and two Impulse Response function (IR) based propagators (Fresnel, Fraunhofer) are implemented and can be selected manually or will be automatically selected based on the critical sampling. (g) Significant extension of phaseqt plotting and statistics capabilities, for instance plotting of Stokes parameters, height errors or unwrapped phases.

The PHASE package contains a selection of scripts for $I D L^{8}$. The functionality is integrated in an $I D L$ class which allows an object-oriented access. The class has a comprehensive inline documentation and provides a great variety of

\footnotetext{
${ }^{1}$ The installation works on actual Linux distributions but also on Solaris and on High Performance Clusters (HPC), we have recent installations on Scientific Linux, Ubuntu, Suse, Debian and HPC. PHASE uses various external libraries which must be installed in advance to use all features.

${ }^{2} Q t$ : qt-project.org/

${ }^{3} Q w t:$ http://qwt.sourceforge.net/

${ }^{4}$ Optimization is based on Minuit2 as part of Root: http://root.cern.ch

${ }^{5}$ Open MPI: http://www.open-mpi.org/

${ }^{6}$ HDF5: http://www.hdfgroup.org/HDF5/

${ }^{7} \mathrm{fftw}:$ http://www.fftw.org/

${ }^{8}$ IDL: http://www.exelisvis.com/ProductsServices/IDL.aspx
} 
functions for electrical field generation, field propagation, field modulation and field import/export, visualization and statistics. To mention some of them: interactive or script oriented propagation with Fourier optics propagators, HDF5 input/ output with auto-detection of various structures, Gaussian and other artificial sources, apertures, slits, mirrors, compound refractive lenses (CRL), phase-unwrapping, plotting etc.. Scripts for the propagation of time dependent pulses are under development. The complete time dependent pulse is represented in a single HDF5 file. Unpacking the time slices, Fourier transformation to frequency slices, propagation of the frequency slices, back-transformation to time slices and packing will be realized from a script.

The PHASE modules use as common interface a simple ascii file ${ }^{\star}$.phase which describes the beamline or optical system. The file may contain references to other data files for sources or height errors. For all used file formats downward compatibility to read old data files has fully been maintained when implementing new features. Writing occurs always in the actual file format.

\section{SLOPE ERROS}

\section{Theory}

A new method to consider the slope errors of the OE's has been implemented to PHASE. In the past PHASE was only capable to consider the surface errors by fitting an $8^{\text {th }}$ order polynomial function to the surface profile of an OE [10]. Such method is sufficient to consider surface errors with low spatial frequencies, but the high frequency part had to be neglected. In an alternative method a phase screen is inserted in an auxiliary plane just behind the mirror, where the phases are obtained by the projection of the surface profile onto this auxiliary plane. This method has been used for instance by [15] [16]. In this method a linear relation between the beam spatial coordinates in the $\mathrm{OE}$ and in the auxiliary plane is assumed. A similar and more sophisticated method uses ray tracing to obtain the phase shifts and has been described in [17]. It is also shown in [17] that the relation between the OE coordinates and the auxiliary plane coordinates can be non-linear.

In PHASE we use a new approach where the surface height value in the position of the critical points $h\left(w_{o}, l_{o}\right)$ is used to determine an additional phase shift of the electric field. Mathematically, the phase shift is introduced by a phase factor outside of the integral of Eq.3. The phase term has the form $e^{i \Delta \Phi}$ where $\Delta \varphi\left(w_{0}, l_{0}\right)=k \cdot \Delta P L=\frac{2 \pi}{\lambda} h\left(w_{0}, l_{0}\right)$. $(\cos (\alpha)+\cos (\beta))$. The height value $h\left(w_{0}, l_{0}\right)$ is the difference between the real and the ideal surface of the $\mathrm{OE}$ and $\triangle P L$ is the additional path length due to $h\left(w_{0}, l_{0}\right)$.

This new approach is similar to those methods using a phase screen in the sense that the surface errors are also converted to phase shifts. This means that the surface errors have no effect in the determination of the PL, which remains the same as for an ideal surface. The error in the calculation of the $E\left(y^{\prime}, z^{\prime}\right)$ in Eq.2 is discussed in more details in the end of this section. It is important to mention, that on top of this the low frequency errors still can be modelled with a high order polynomial.

Different from the phase screen method, in the new scheme as implemented in PHASE the coordinates on the OE and the coordinates in the image plane are already well defined. This permits a simple introduction of a phase error term to each individual CP-contribution in Eq.3. Moreover, a proper implementation of the phase factor does not cause a significant increase of computational time as compared to an ideal surface.

When propagating beams with the new method the positions of the source plane and the image plane have to be chosen carefully. For ideal surfaces and $2^{\text {nd }}$ order SPA the only constraint for SP and IP results from the requirement that the critical points have to be far apart and, thus, an analytic integration to infinity is justified. Including slope errors a further constraint has to be regarded. The new method assumes a constant height error in the "surrounding area" of the critical point. The surrounding area is understood as the region of the OE surface around a critical point where the function $\cos (k \cdot P L)$ oscillates slowly. The major contribution to the integral in Eq.3 arises from this area. The size of surrounding area must be adapted to the spatial variation of the height errors around a critical point as illustrated in Fig.9, where the function $\cos (k \cdot P L)$ is plotted versus $w$, together with its integral value for some different conditions. Obviously, the size of surrounding area is dependent upon the choice of SP and IP. This will be discussed in more detail in the following. 

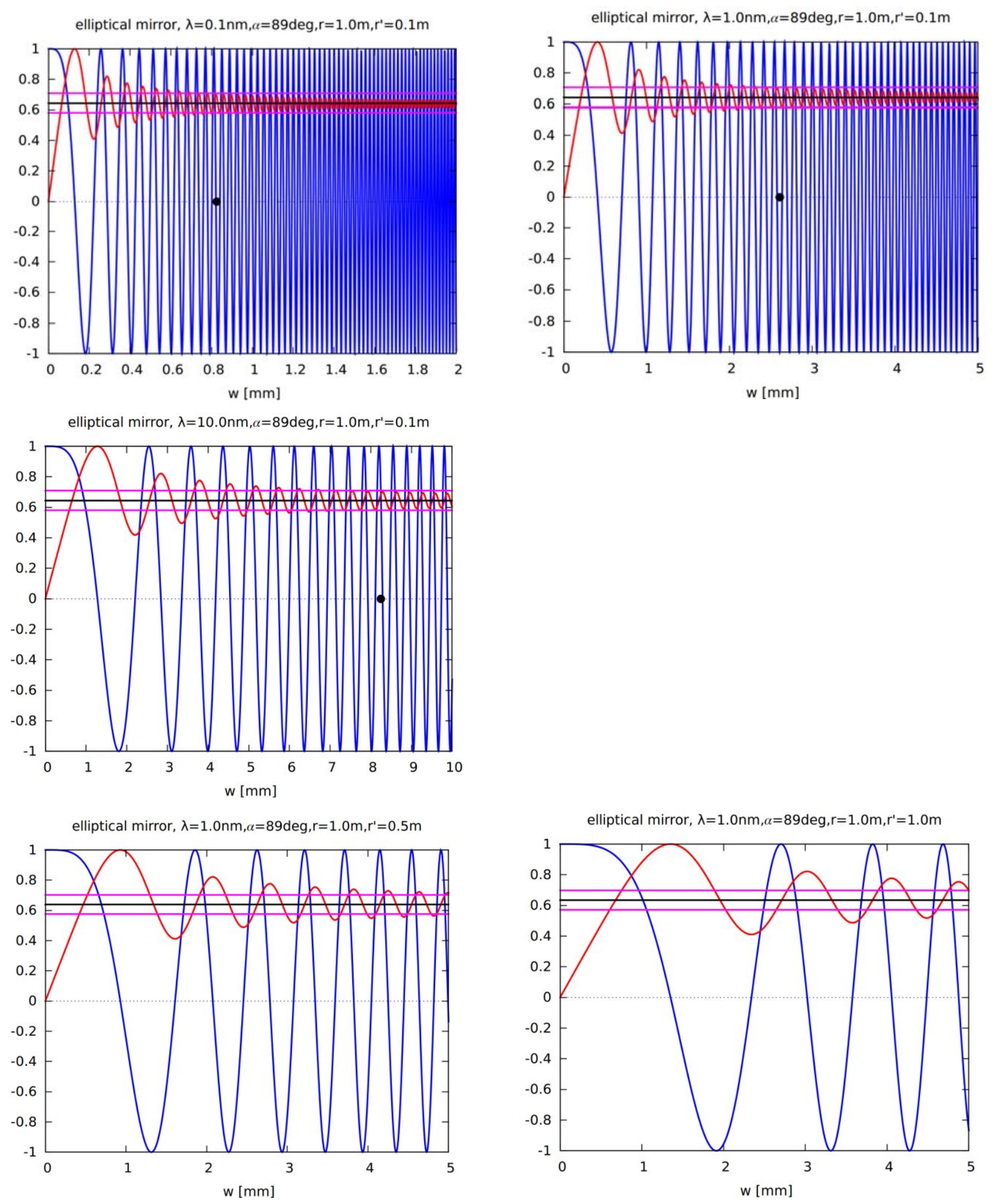

Figure 9a-e: Blue curves: $\cos (k \cdot P L)$; red curves: $\int_{0}^{\infty} \cos (k \cdot P L) \cdot d w$; black lines: theoretical values of $\int_{0}^{\infty} \cos (k \cdot P L) \cdot d w=$ $\sqrt{\pi /\left(2 k \cdot P L_{w w}\right)}$; magenta curves: $(1 \pm 0.1) \cdot \sqrt{\pi /\left(2 k \cdot P L_{w w}\right)}$, showing where the oscillations of the integral is within a interval of $10 \%$ of the theoretical value. The point with the black marker is the value of $w_{10}$ calculated with Eq.20. For all the graphs: elliptical mirror, focal planes at $10 \mathrm{~m}$ and $1 \mathrm{~m}$ (10:1 demagnification), $\alpha=89 \mathrm{deg}, \mathrm{r}=1 \mathrm{~m}$. See the values of wavelength and $\mathrm{r}, \mathrm{r}$ ' on the titles. 
It can be noted that after a certain number of oscillations of $\cos (k \cdot P L)$ the value of the integral converges within a certain interval. We can thus (arbitrarily) define the surrounding area as the region where the integral of $\cos (k \cdot P L)$ oscillates at values bigger than $\pm 10 \%$. For the cases presented here, this occurs within the region of the first ten oscillations. The value of $w$ after ten oscillations $w_{10}$ (i.e. the value of the $20^{\text {th }}$ root of the function $\cos (k \cdot P L)$ can then be used to compare the size of the surrounding area at different conditions. Fig.9 a-c show that the values of $w_{10}$ are in the interval between $\sim 1 \mathrm{~mm}$ and $\sim 10 \mathrm{~mm}$ in the wavelength range from $1 \AA$ to $10 \mathrm{~nm}$. This means for the integration of Eq.3, that an entire region of few millimeters around the critical point is assumed to have the same height error of the critical point.

Some analytical results for the value of $w_{10}$ can be derived by using some approximations. First, by assuming that the $P L$ in Eq.5 has only a quadratic dependence in $w$, we can derive that

$$
\frac{2 \pi}{\lambda} \frac{P L_{w w}}{2} w_{10}^{2}=\left(n+\frac{1}{2}\right) \pi
$$

and

$$
w_{10}=\sqrt{(n+0.5) \frac{\lambda}{P L_{w w}}}
$$

where $\mathrm{n}=20$ for the $20^{\text {th }}$ zero of $\cos (k \cdot P L)$. The expression for $P L_{w w}\left(z, y, z^{\prime}, y^{\prime}, w, l, r, r^{\prime}, \alpha, \beta\right)$ is in general complicated for elliptical surfaces. But it becomes simple in the case of a toroidal mirror at distances $z=y=z^{\prime}=y^{\prime}=w=l=0$ and $\beta=-\alpha$. At this condition the expressions for $P L_{w w}$ reduces to

$$
P L_{w w}\left(0,0,0,0,0,0, r, r^{\prime}, \alpha,-\alpha\right)=\cos (\alpha)^{2}\left(\frac{1}{r^{\prime}}+\frac{1}{r}\right)-\frac{2 \cos (\alpha)}{R+\rho}
$$

where $R$ and $\rho$ are the radii of the toroidal mirror. Although this seems a very particular result, this expression can be used to understand the general dependences of $P L_{w w}$ and $w_{10}$ for a general surface. Combining Eq.21 and Eq.22 we obtain that $w_{10}$ has a dependence of $w_{10} \sim \sqrt{\lambda}$ and that it is smaller for smaller values of $r$ and $r^{\prime}$. From this we conclude that the current method yields better results for small wavelengths and by using source and image planes close to the OE, that is for $r \rightarrow 0$ and $r^{\prime} \rightarrow 0$. These dependences are illustrated in Fig.9, where in $a-c$ we see the dependence of $w_{10}$ with the wavelength, whilst Fig.9b, $d$ and $e$ show the dependence upon $r$.

The effect of the surface errors in the value of the integral in Eq.3 can be evaluated by comparing the values of $I_{1}=\int_{-\infty}^{\infty} \cos (k \cdot P L) \cdot d w=\sqrt{\frac{\pi}{k \cdot P L_{w w}}}$ and of $I_{2}=\int_{-\infty}^{\infty} \cos (k \cdot P L(w)+k \cdot \Delta P L) \cdot d w$. In Fig.10 we compare some real error profiles $h(w, l)$ with the function $\cos (k \cdot P L)$.
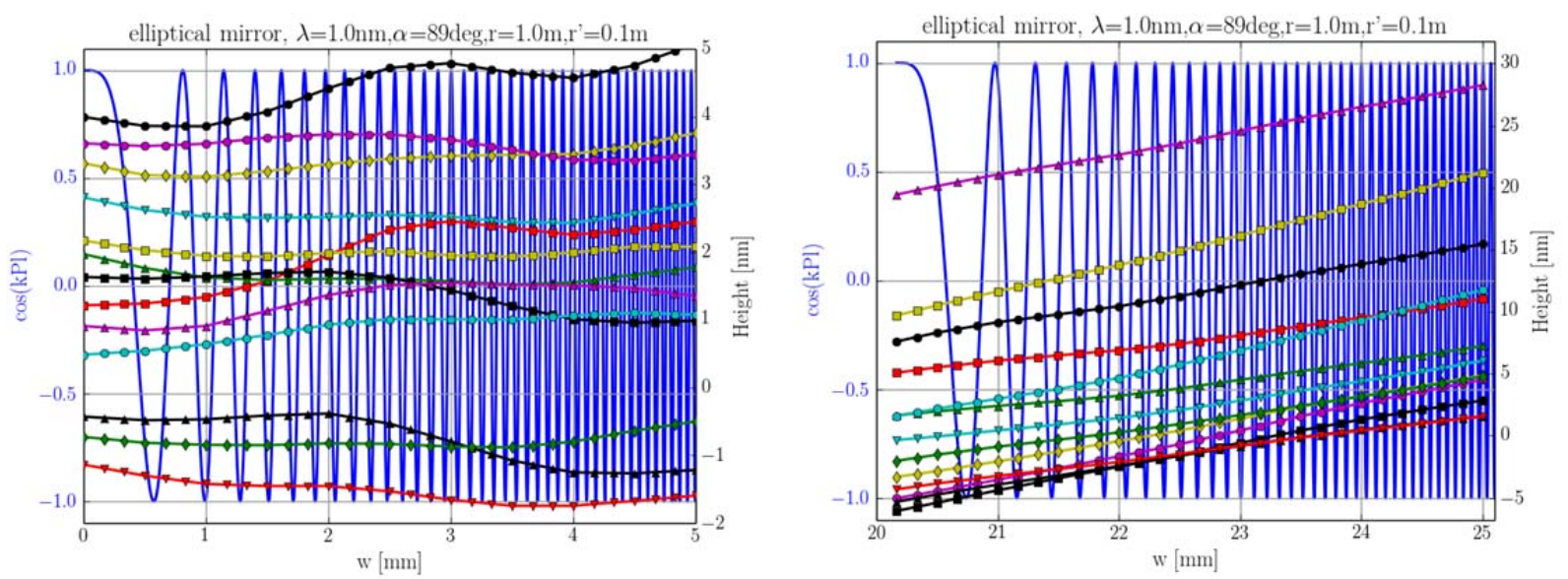

Figure 10: Blue curves: $\cos (k \cdot P L)$. Optical values on the titles. The points show the height values of the same profiles of Fig.16a and $\mathrm{b}$, respectively, profiles in $l$ in the interval $\pm 6 \mathrm{~mm}$ in steps of $1 \mathrm{~mm}$. 
In those graphs we see a smooth variation of $h(w, l)$ within the region of $w<w_{10}$. This permits the assumption of a linear dependence of $h(w)$ on $w$ in the form of $h(w)=h_{w} \cdot w+h_{0}$, where $h_{w}=\partial h(w) / \partial w$. Thus, in the second order approximation of the path length, we obtain

$$
P L(w)+\Delta P L(w)=\frac{P L_{w w}}{2} w^{2}+2 \cos (\alpha) h(w)=\frac{P L_{w w}}{2} w^{2}+2 \cos (\alpha) h_{w} w+2 \cos (\alpha) h_{0}
$$

where the constant term $2 \cos (\alpha) h_{0}$ can be dropped for the discussion because it is already included in the phase term $e^{i \Delta \Phi}$. With these approximations the integral $I_{2}$ results in

$$
I_{2}=\int_{-\infty}^{\infty} \cos (k P L+k \Delta P L) \cdot d w=\frac{\sqrt{\pi}}{\sqrt{k P_{w w}}}\left[\sin \left(\frac{2 \cos (\alpha)^{2} k h_{w}^{2}}{P L_{w w}}\right)+\cos \left(\frac{2 \cos (\alpha)^{2} k h_{w}^{2}}{P L_{w w}}\right)\right]
$$

Comparing the integrals $I_{1}$ and $I_{2}$ we have $I_{1} \approx I_{2}$ for

$$
\varepsilon=\frac{2 \cos (\alpha)^{2} k h_{w}^{2}}{P L_{w w}} \ll 1
$$

By using the numerical values of Fig.10, we obtain for those examples $\epsilon=0.00126$ for $h_{w}=1 \mathrm{~nm} / \mathrm{mm}$ (Fig.10a), and $\epsilon=$ 0.0317 for $h_{w}=5 \mathrm{~nm} / \mathrm{mm}$ (Fig.10b), where this latter value represents a typical poor quality optical surface. Finally, the error in the calculation of $E\left(y^{\prime}, z^{\prime}\right)$ for $\epsilon<<1$ is given by $\left(I_{1}-I_{2}\right) / I_{1}=-\varepsilon$. Again we observe that bigger values of $P_{w w}$ produce more accurate results. Moreover, from these numerical results we conclude that even poor quality surface would have a small effect in the result of the integration in Eq.3. The same comparison done above was done to the $\sin (k \cdot P L)$ part of the integral and the results are consistent to the ones presented here.

\section{Numeric Tests and Examples}

The validity of the method proposed here was studied by simulating a plane mirror where the surface error $h(w, l)$ has a linear dependence with the mirror coordinate $w$ given by $h(w, l)=\delta \cdot w$. This particular error profile is similar to an ideal plane mirror (i.e. no surface error) with an angular misalignment of $-\delta$ radians around the axis $l$ in Fig. 1 . Since PHASE can perform calculations considering misaligned OE's, we can therefore compare the results of the simulations of the linear surface error with the results of a misaligned mirror. For these simulations was used a simple circular source with a plane wavefront. To avoid diffraction artefacts in the results we used a source with smooth edges (instead of a source defined by a plane wave trough an aperture). In the test cases we modelled a source with a spatial distribution defined by a Fermi-Dirac like distribution of the form $E(y, z)=1 /\{1+\exp [(r-0.5 E-3) / 5 E-5]\}$, where $r=\operatorname{sqrt}\left(y^{2}+z^{2}\right)$. This results in a circular source with $1 \mathrm{~mm}$ of diameter (FWHM) and a smooth edge, as shown in Fig.11. The source and image plane are placed $1 \mathrm{~m}$ before and after the plane mirror, which operates at $\alpha=88 \mathrm{deg}$. The resulting phase values of the image for the ideal surface are shown in Fig. 12 for comparison.

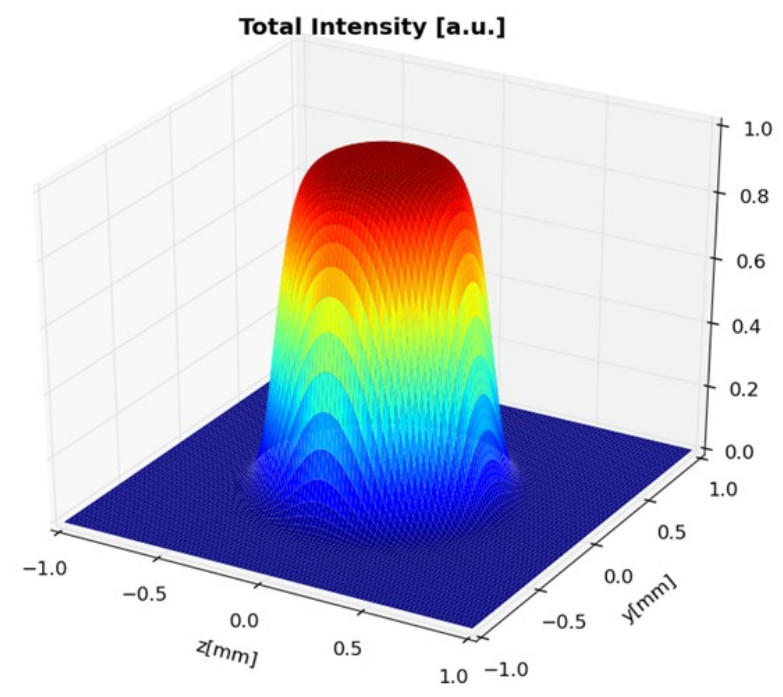

Figure 11: Source intensity spatial distribution for the test cases. 

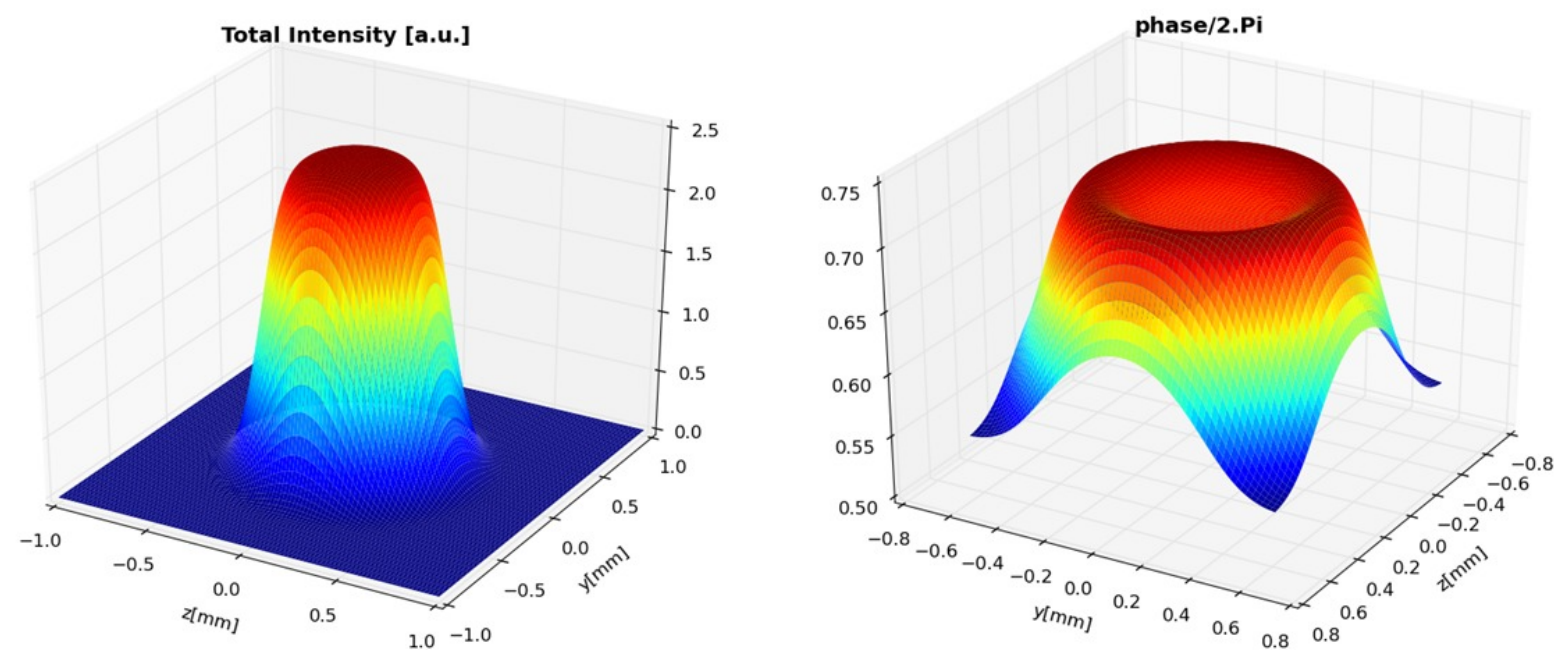

Figure 12: Test case results for an ideal surface(no surface error and no misalignment): (a) intensity and (b) phase values in the image plane. All the graphs of phase show only the values for points which the corresponding intensity is bigger than $1 \%$.

Several values of $\delta$ have been simulated. Here we discuss the cases of $\delta$ equal to $300 \mathrm{nrad}, 1 \mu \mathrm{rad}$ and $5 \mu \mathrm{rad}$. The phase values for the case $\delta=1 \mu \mathrm{rad}$ are shown in Fig.13, where we clearly see the tilt of the wavefront.

The comparison with the simulation of a misaligned mirror is shown in Fig.14 for the profile taken at $\mathrm{z}^{\prime}=0$. We observe for the $1 \mu \mathrm{rad}$ case a maximum difference in intensity (blue curve) of $\sim 1 \%$ of the maximum intensity value whereas the difference in the phase values is of $\sim 1 \%$ in the central part and of $\sim 5 \%$ on the borders. If we consider that the footprint of the beam on the mirror surface has a size of $1 \mathrm{~mm} / \cos \left(88^{\circ}\right)=28.6 \mathrm{~mm}$, then, the borders of the beam are at $w= \pm 14.3 \mathrm{~mm}$ and thus the surface errors $h$ at the borders are $h= \pm 1 \mathrm{e}-6 * 14.3 \mathrm{e}-3 \approx \pm 15 \mathrm{~nm}$. From this we can estimate that for the current method and geometry the height error must be smaller than $\approx 10 \mathrm{~nm}(20 \mathrm{~nm}$ peak-to-valley) in order to have results with a difference of roughly $1 \%$. For $\delta=5 \mu \mathrm{rad}$ the differences are of $10 \%$ for both intensity and phase values (Fig.14). And finally for $\delta=300$ nrad the phase difference at the borders reach $2 \%$ and the differences of intensities are smaller than $0.5 \%$ (Fig. 14 ).

A final test was done by propagating the results for $\delta=1 \mu \mathrm{rad}$ by $100 \mathrm{~m}$. It is expected that the peak value is displaced by $200 \mu \mathrm{m}$. This displacement is observed in the simulations (Fig.15) in agreement with the results obtained with a misaligned surface.

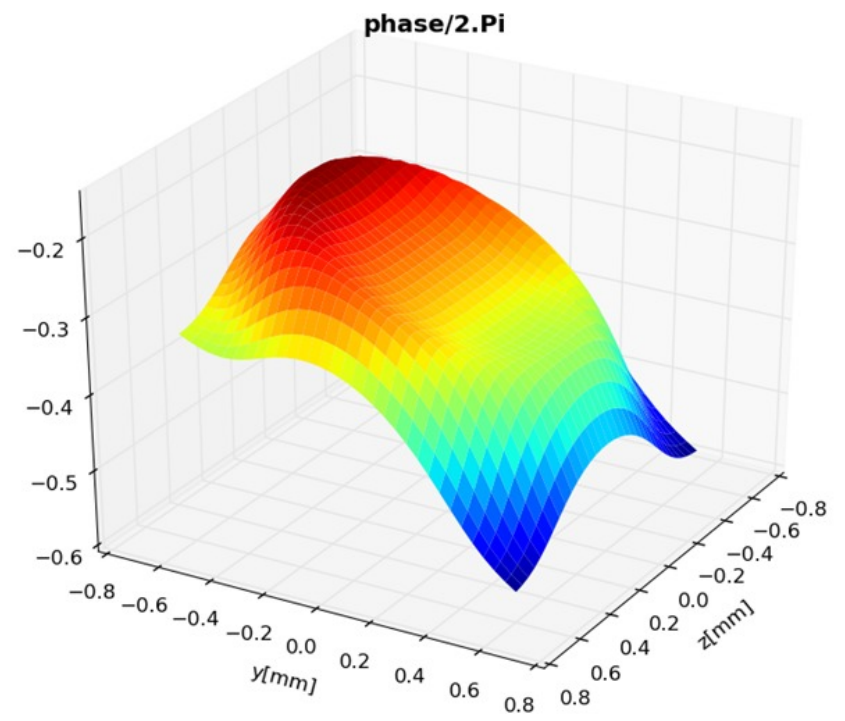

Figure 13: Phase for the test case $\delta=1 \mu \mathrm{rad}$. 

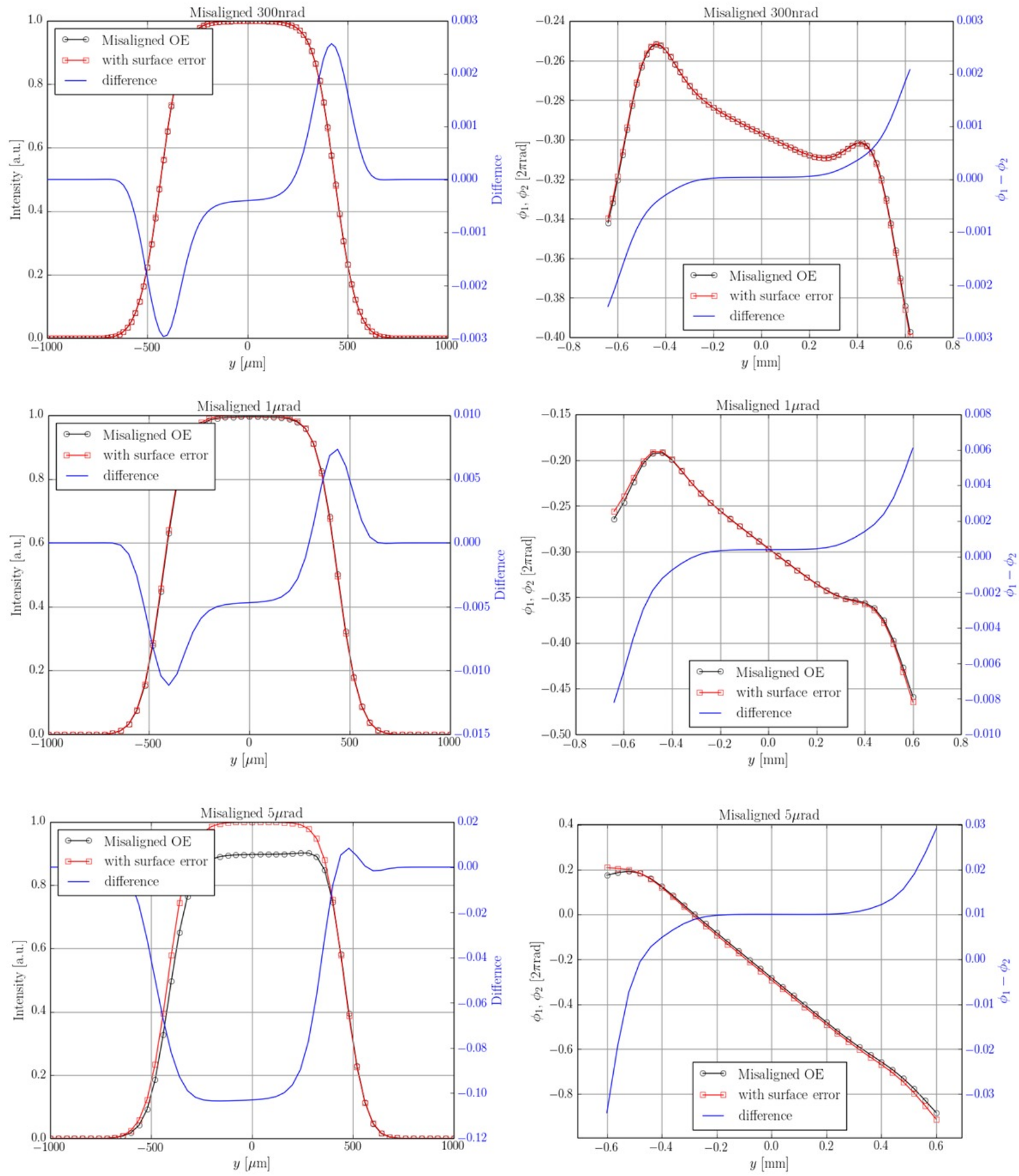

Figure 14: Results of the test cases comparing the results with slope error $\mathrm{h}(\mathrm{w})=\delta \mathrm{w}$ and misaligned surface. Top: $\delta=300 \mathrm{nrad}$; center: $\delta=1 \mu \mathrm{rad}$; bottom: $\delta=5 \mu \mathrm{rad}$. Left column: comparison of intensities; right column: comparison of phases. Red: simulation with the new slope error functionality; black: simulation with the misalignment feature of PHASE; blue: difference of red and black curves. 


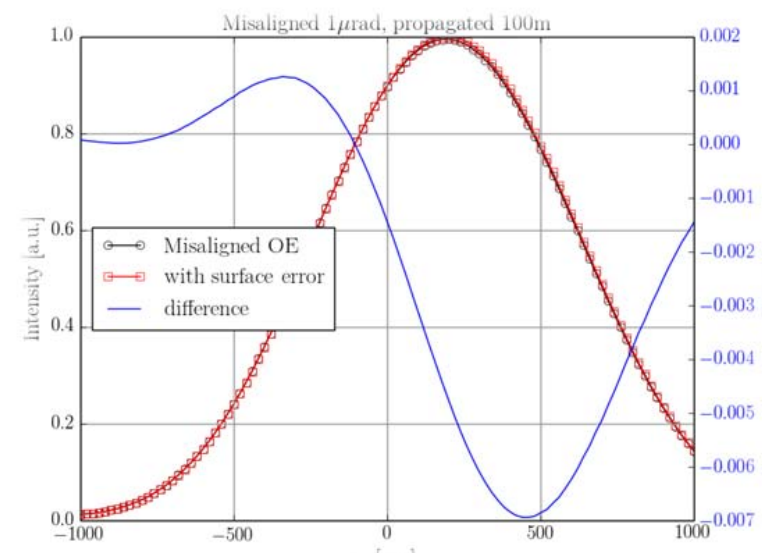

Figure 15: Result for $\delta=1 \mu \mathrm{rad}$ propagated by $100 \mathrm{~m}$, where is observed a peak of intensity at $\mathrm{y}=200 \mu \mathrm{m}$, as expected.

Finally, we used a height error profile from a real mirror for beam propagation. Two profiles are used as examples for a good and a poor quality surface (Fig.16 left (a) and right (b), respectively). The data have been taken at the Helmholtzzentrum Berlin with the BESSY-NOM [18]. The local curvature of the measured elliptical mirror ranges from $6 \mathrm{~m}$ to $16 \mathrm{~m}$. The measurement accuracy accompanied with these variations is of the order of $1 \mathrm{~nm} \mathrm{rms}$. These height errors were used as the surface error for a hypothetic elliptical mirror in a 10:1 demagnification geometry operating at $\alpha=89 \mathrm{deg}$. A Gaussian source with beam waist of $50 \mu \mathrm{m}$ and $\lambda=10 \mathrm{~nm}$ was used. The image plane was placed $10 \mathrm{~mm}$ behind the focus to highlight the artefacts in the image due to the surface errors. The results are presented in Fig. 17 and Fig. 18 in comparison with results of the ideal surface. These graphs illustrate the new capability of PHASE.
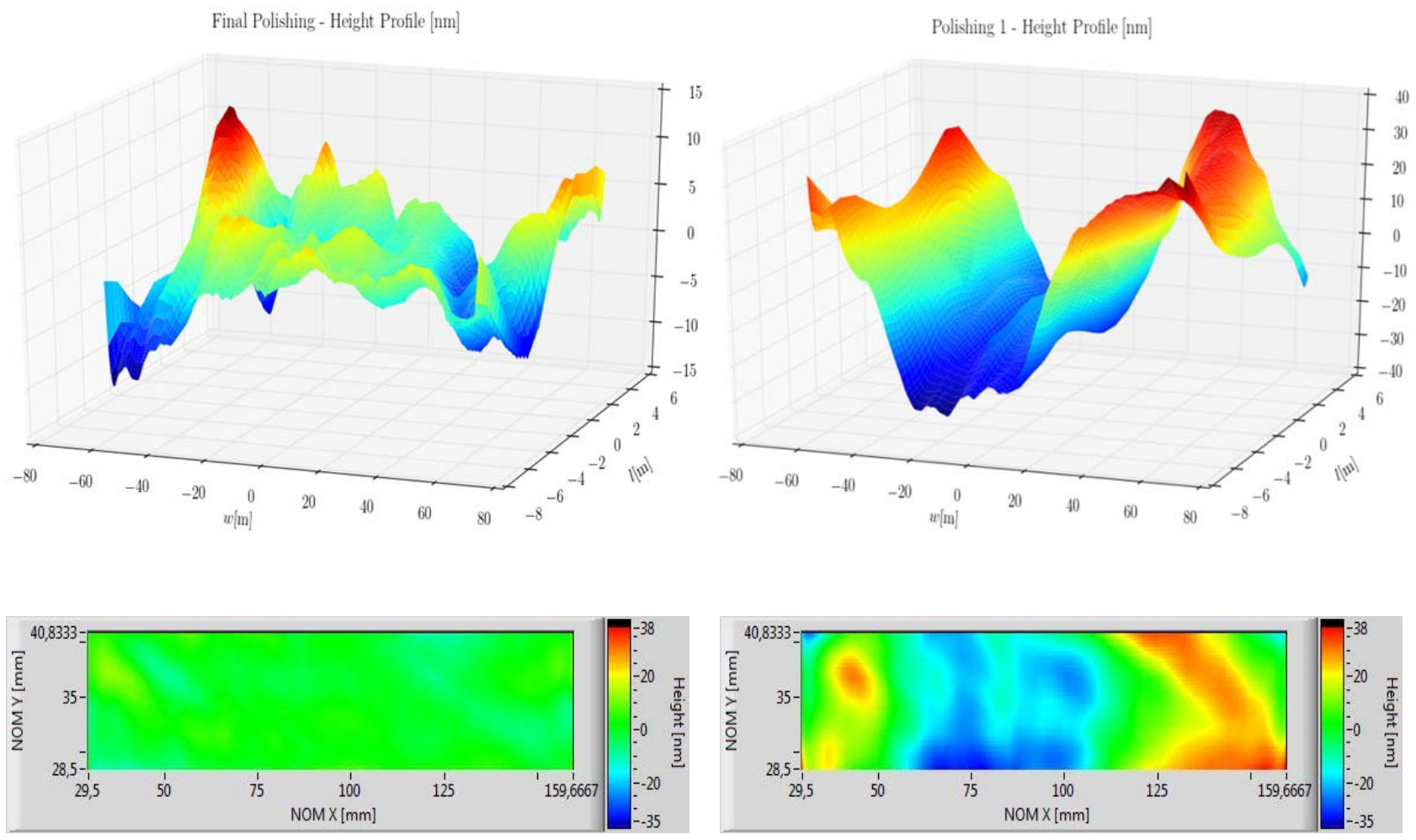

Figure 16: Real surface error profiles in $\mathrm{nm}$ as used in the simulations. These are examples of a good (left, a) and poor (right b) quality surfaces. The data are actually from the same mirror where surface (a) is the resulting surface after the final polishing and (b) is the profile before the final polishing. The data is the difference between the real and the ideal surface. 

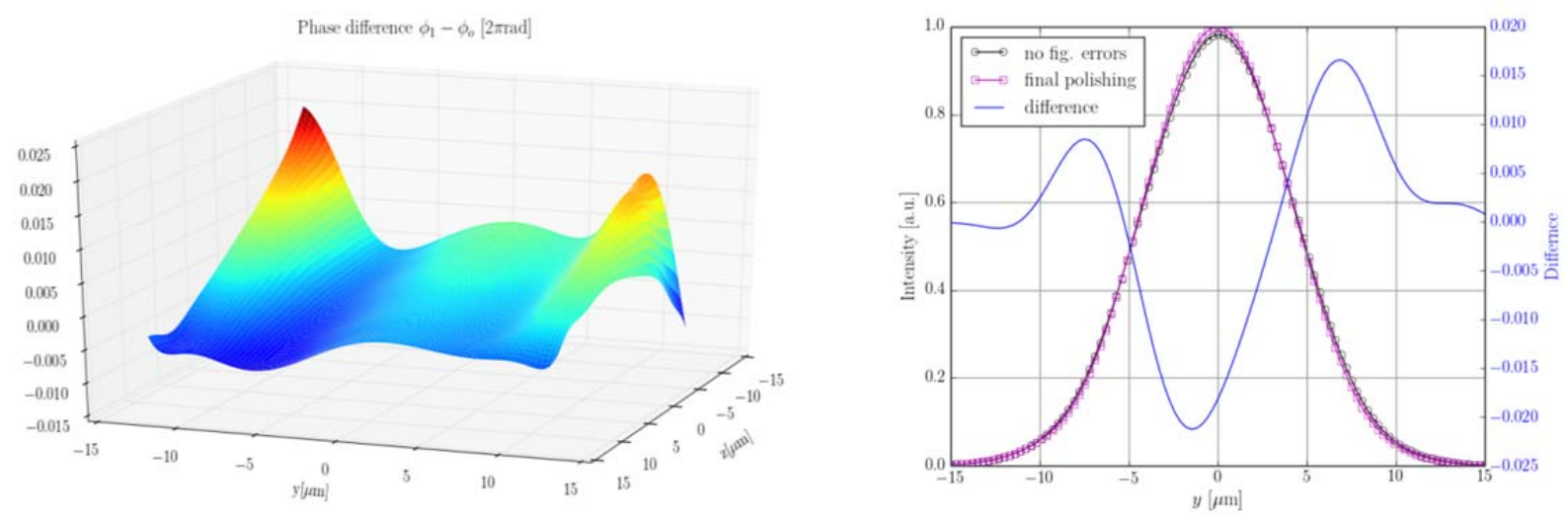

Figure 17: Results of simulations considering an elliptical mirror in a 10:1 geometry with the surface errors of Fig.16a. Left: Difference of the phases for simulations with and without slope errors. Right: Difference of intensities.
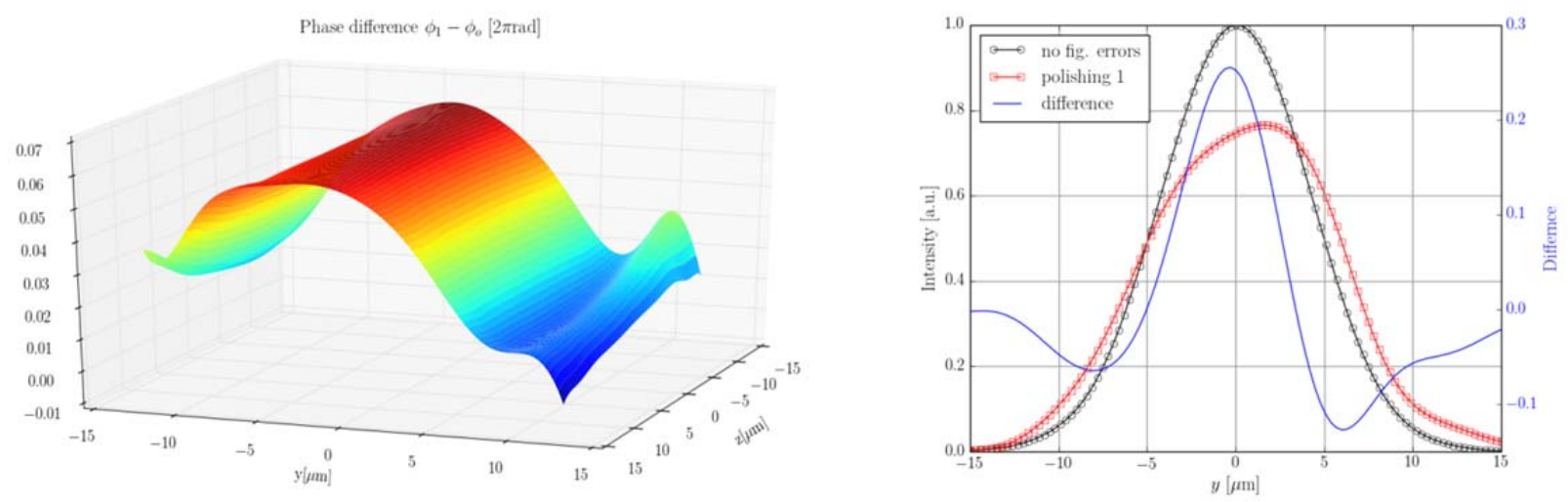

Figure 18: Same as Fig.17 for the profile of Fig.16b.

\section{CONCLUSION}

The new developments of the PHASE package have been presented. The accuracy of the $2^{\text {nd }}$ order stationary phase approximation was studied in detail and it was concluded that the best results are obtained when the source and the image planes are located close to the optical element. Short range slope errors were implemented into PHASE and tested. Flat error profiles are assumed in a certain region around the critical points. The size of these regions is in the order of a few $\mathrm{mm}$. The size is minimized when the source and image planes are at short distances to the optical element. Apertures can easily be implemented as independent objects and if beam scraping by the optical element is important an equivalent aperture (oriented perpendicular to the beam) should be located shortly behind the OE. Mathematically, there is a freedom in choosing the exact location of the source and the image plane. All results of this article, however, strongly favor locations close to the OE. The Fourier Optic propagators included in the PHASE package will support an easy propagation of a source to the starting position and the results to the final position.

\section{REFERENCES}

[1] F. Cerrina, Proceedings of SPIE 503, 68-77 (1984).

[2] M. Sanchez del Rio, N. Canestrari, F. Jiang and F. Cerrina, "SHADOW 3: A new version of the synchrotron X-ray optics modelling package," Journal of Synchrotron Radiation 18, 708-716 (2011). 
[3] F. Schäfers, "Modern Developments in X-Ray and Neutron Optics in Springer Series in Optical Science Ed. A. Erko et al.," Berlin Heidleberg Springer-Verlag 137, 9 (2008).

[4] P. Emma et al., "First lasing and operation of an angström-wavelength free-electron laser," Nature Photonics 4, 641647 (2010).

[5] T. Tanaka and M. Yabashi et al., "A compact X-ray free-electron laseremitting in the sub-angström-region," Nature Photonics 6, 540-544 (2012).

[6] S. Leemann et al., "Beam dynamics and expected performance of Sweden's new storage-ring light source: MAX IV," Physical Review Special Topics - Accelerator and Beams, 12, 120701 (2009).

[7] J. Bahrdt, "Fourth Order Optical Aberrations and Phase Space Transformation for Reflection and Diffraction Optics," Applied Optics 34 (1), 114-127 (1995).

[8] J. Bahrdt, "Wave Front Propagation: Design Code for Synchrotron Radiation Beamlines," Applied Optics 36 (19), 4367-4381 (1997).

[9] J. Bahrdt, "Wavefront Tracking Within the Stationary Phase Approximation," Physical Review Special Topics Accelerator and Beams 10, 060701 (2007).

[10] J. Bahrdt, U. Flechsig, S. Gerhardt and I. Schneider, "PHASE, a Universal Software Package for the Propagation of Time-Dependent Light Pulses along Grazing Incidence Optics," Proceedings of SPIE Advances in Computational Methods for X-Ray Optics II 8141, 13 (2011).

[11] L. Mandel, E. Wolf, "Optical Coherence and Quantum Optics," Cambridge England: Cambridge University Press, (1995).

[12] J. Focke, "Asymptotische Entwicklungen mittels der Methode der stationären Phase," Berichte über die Verhandlungen der sächsischen Akademie der Wissenschaften zu Leipzig; Mathematisch Naturwissenschaftliche Klasse 101 (3), 1-48 (1954).

[13] B. Henke, E. M. Gullikson and J. Davis, "X-ray interactions: photoabsorption, scattering, transmission and reflection at E=50-30000eV, Z=1-92," Atomic Data and Nuclear Data Tables 54, 181-342 (1993).

[14] S. Reiche, "GENESIS 1.3: a fully 3d-time-dependent FEL simulation code," Nuclear Instruments and Methods in Physics Research A 429, 243-248 (1999).

[15] L. Samoylova, H. Sinn, F. Siewert, H. Mimura, K. Yamauchi and T. Tschentscher, "Requirements on Hard X-Ray Grazing Incidence Optics for the European XFEL: Analysis and Simulation of Wavefront Transformations," AIP Conference Proceedings of SPIE 7360, 73600E (2009).

[16] M. Fuchs, R. Sweet, L. Berman, W. Hendrickson, O. Chubar, N. Canestrari, M. Idir, L. Yang and D. Schneider, "NSLS-II Biomedical Beamlines for Macromelecular Crystallography, FMX and AMX, and for X-Ray Scattering, LIX: Current Developments," IOP Conference Proceedings 493, 012021 (2014).

[17] X. Shi, R. Reininger, M. Sanchez del Rio and L. Assoufid, "A Hybrid Method for X-Ray Optics Simulation: Combining Geometric Ray-Tracing and Wavefront Propagation," Journal of Synchrotron Radiation 21,1 (2014).

[18] F. Siewert, et al., "Sub-nm accuracy metrology for ultra-precise reflective X-ray optics," Nuclear Instruments and Methods in Physics Research A Supplement I 635, 52-57 (2011). 